Research Article

\title{
Impact of SARS-CoV-2 Outbreak on Physical and Mental Health, Psychological Distress, and Suicidal Ideation, and the Mediating Role of Stressors
}

\author{
Keita Kiuchi ${ }^{1,}{ }^{*}$, Katsumasa Kishi ${ }^{2}$, Kanto Araki ${ }^{3}$
}

1. Research Center for Overwork-Related Disorders, National Institute of Occupational Safety and Health, Kawasaki, Japan; E-Mail: kiuchi@h.jniosh.johas.go.jp

2. Practical Psychology Institute, LLC, Kawasaki, Japan; E-Mail: jwqcj020@yahoo.co.jp

3. Center for Asian and Pacific Studies, Seikei University, Musashino, Japan; E-Mail: araki.kanto@ejs.seikei.ac.jp

* Correspondence: Keita Kiuchi; E-Mail: kiuchi@h.jniosh.johas.go.jp

Academic Editor: Soo Liang Ooi

Special Issue: Complementary, Traditional, and Integrative Medicine for COVID-19

OBM Integrative and Complementary Medicine

2021, volume 6, issue 1

doi:10.21926/obm.icm.2101010
Received: December 23, 2020

Accepted: March 10, 2021

Published: March 17, 2021

\begin{abstract}
This study investigated the physical and psychological impacts of elements related to coronavirus disease (COVID-19) and demographic data in Japan both directly and as mediated by stressors, immediately following the rescinding of the state of emergency declaration accompanying the severe acute respiratory syndrome coronavirus 2 outbreak. An online survey was administered to 1,500 Japanese registered with an online outsourcing service. Data were analyzed with structural equation modeling using Mplus. Relatively large total effects were observed for "insufficient exercise" on physical health ( $B=-0.24, S E=0.03)$, "mental health issues related to COVID-19" on mental health $(B=-0.25, S E=0.03)$ and psychological distress $(B=0.24, S E=0.03)$, neuroticism on mental health $(B=-0.30, S E=0.02)$ and psychological distress $(B=0.36, S E=0.02)$, and social support on suicidal ideation $(B=-$ $0.32, S E=0.03)$. Thus, mitigating the harm resulting from the ongoing impact of the COVID-
\end{abstract}

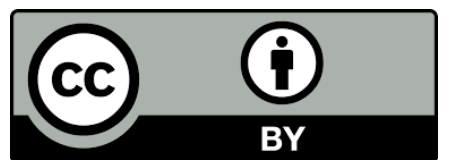

(c) 2021 by the author. This is an open access article distributed under the conditions of the Creative Commons by Attribution License, which permits unrestricted use, distribution, and reproduction in any medium or format, provided the original work is correctly cited. 
19 infection outbreak by raising awareness of risk factors and promoting social support is feasible.

\section{Keywords}

COVID-19; SARS-CoV-2; mental health; physical fitness; psychological distress; suicidal ideation; mediation; stressor; coping; community

\section{Introduction}

Studies have been published in many countries regarding the psychological impact of the severe acute respiratory syndrome coronavirus 2 (SARS-CoV-2) outbreak. An early-stage review of four cross-sectional studies from China suggests that anxiety, depression, stress, and sleep disorders may be observed during the coronavirus disease (COVID-19) pandemic [1]. Following this review, there have been several studies reporting the results of general public surveys. For example, a large-scale study from the United States found that fear was particularly high in areas with many reported cases of COVID-19, and observed a correlation between being socially vulnerable (female, Asian, Hispanic, born abroad, family with children) and fear [2]. Also, longitudinal studies from China and Australia initiated before the SARS-CoV-2 outbreak demonstrated the occurrence of psychological responses owing to the outbreak $[3,4]$. Further, serum 25-hydroxyvitamin D concentrations, assumed to be related to mental health [5], have also been reported to be decreased in patients with emotional disorders under extended COVID-19 infection [6].

The negative impact of the SARS-CoV-2 outbreak on mental health is gradually becoming clear. Thus, the current challenge for research is the determination of the elements impacting these negative psychological responses. Further, the risk of suicide, which is the worst possible psychological response in a disaster situation, is unclear.

Considering a stress model of psychological response during a disaster suggests that social support and coping style can influence the psychological response level. Studies have shown that social support reduces anxiety and depression amid the SARS-CoV-2 outbreak $[7,8]$. Negative thinking and avoidant behavior accompanying negative coping have been reported to be associated with depression and psychological distress $[9,10]$.

Although there is limited research about the SARS-CoV-2 outbreak, personality traits may also impact a stress response. A past meta-analysis found a negative association between neuroticism and mental health [11]. As for predictors of psychological distress, cyclothymic, depressive, and anxious temperaments in personality with a need for approval in attachment style were reported as risk factors and confidence and discomfort with closeness in attachment style as protective factors [12]. Further, people with high extraversion experience have been hypothesized with greater psychological stress amid circumstances limiting social interaction, such as the SARS-CoV-2 outbreak.

People cannot live without stressors, the factors that produce mental and physical stress responses. Excessive exposure to stressors can lead to hyper and chronic stress reactions, which in turn can worsen physical and mental health-related outcomes such as physical and mental deterioration, psychological distress, and suicidal ideation. In contrast, appropriate coping can reduce perceived stress and improve physical and mental health. Such an association between 
stressors, coping, and physical and mental health outcomes have also been reported under the influence of COVID-19 [13, 14]. Even under the spread of COVID-19 infection, appropriate coping will likely have a positive effect, both directly through the improvement of physical and mental health-related outcomes and indirectly through the reduction of perceived stress on stressors such as unemployment.

From the aforementioned discourse, it is clear that there is insufficient detailed research on psychological responses to the SARS-CoV-2 outbreak in the general public focusing on the Japanese population. Moreover, many studies from other countries and regions have used adjusted correlations between variables; however, only a few focused on mediated correlations between variables. Thus, this study aimed to investigate both the direct and stressor-mediated impact of demographic data, elements related to COVID-19 infection, personality, social support, and coping on physical and psychological status using structural equation modeling (SEM).

\section{Materials and Methods}

\subsection{Participants}

An online survey was administered to the Japanese who were 18 years or older registered with an online outsourcing service provided by Crowd Words, Inc. The responses were received from 1,500 participants between May 26 and May 27, 2020 (viewed by 2,594 individuals, acceptance rate: $57.8 \%)$. Only the participants lacking literacy or cognitive function were excluded. The participants were screened based on responses that were carefully examined to identify random or consistently contradictory responses. No responses fulfilling the exclusion criteria were identified, and all data were used for analysis. More detailed information on the basic characteristics of the participants has been reported in our existing short report [15].

\subsection{Survey Items}

\subsubsection{Basic Demographic Information}

Participants were inquired about the basic demographic information, including age (multiple choices from under 19 years to 70 years or older); sex (female, male, other); prefecture of residence; the highest level of education completed (middle school graduate, high school graduate, junior college/vocational school/technical school graduate, college graduate, graduate school graduate); marital status (unmarried, divorced/widowed, married [do not live with a partner], married [live with a partner]); ethnicity (Asian, black, multi-ethnic, white, Chinese, Middle Eastern/Arab, other); employment status before the outbreak (student, self-employed/sole proprietorship, operating a company, full-time, part-time, contract worker, housewife/househusband, on leave [childcare leave, sick leave, unemployed]); and annual household income before the outbreak (selected from $<18600$ USD, 18600 USD to 37200 USD, 37300 USD to 74500 USD, 74600 USD to 111800 USD, 111900 USD to 149000 USD, and $\geq 149000$ USD).

Regarding the SARS-CoV-2 outbreak, participants were enquired about how often they went to their workplace under the state of emergency declaration (almost never, half the week or less, more than half the week, almost every day); amenities in their residence and the surrounding environment (whether they had a garden, balcony, parks, or botanical gardens); the number of 
preschool-age children in the home; whether they had underlying physical or psychiatric disorders; whether they had been infected with COVID-19 (recovered, recovering, infection suspected, not infected); about contact with infected persons in the past week (had contact, suspected contact, no contact); and extent of voluntary restriction of outside activity (did not leave home at all, did not leave home unless exercising or shopping for food, went out for work in addition to shopping when needed, did not refrain from going out but practiced social distancing, or did not refrain from going out and did not practice social distancing).

Concerning stressors, participants were asked to assess 15 items regarding life events likely to occur under the state of emergency declaration on a four-point scale of "did not happen," "happened, but was not stressful or difficult," "was a little stressful or difficult," or "was very stressful or difficult" (see Table 1 for the list of items). Each item was scored from 0 to 3 points.

Lastly, participants were asked about whether they performed any of the seven intentional behaviors intended to bolster mental health or change the mood to evaluate "coping" (see Table 1 for the list of items).

Table 1 Frequency of life events, stressors, and coping.

\begin{tabular}{|c|c|c|c|c|c|c|c|c|c|}
\hline \multicolumn{2}{|c|}{ Life Events and Stressors } & \multicolumn{2}{|c|}{ Not happen } & \multicolumn{2}{|c|}{ Not stressful } & \multicolumn{2}{|c|}{ A little stressful } & \multicolumn{2}{|c|}{ Very stressful } \\
\hline NO. & Contents & $\mathrm{N}$ & $\%$ & $\mathrm{~N}$ & $\%$ & $\mathrm{~N}$ & $\%$ & $\mathrm{~N}$ & $\%$ \\
\hline E1 & $\begin{array}{l}\text { My household income significantly decreased (by } 40 \% \\
\text { or more). }\end{array}$ & 1022 & 68.1 & 148 & 9.9 & 201 & 13.4 & 129 & 8.6 \\
\hline E2 & $\begin{array}{l}\text { I lost someone close to me (due to the novel } \\
\text { coronavirus or other causes). }\end{array}$ & 1393 & 92.9 & 39 & 2.6 & 41 & 2.7 & 27 & 1.8 \\
\hline E3 & $\begin{array}{l}\text { I had difficulty getting a consultation with a doctor for } \\
\text { the treatment of a chronic disease I have. }\end{array}$ & 1190 & 79.3 & 140 & 9.3 & 137 & 9.1 & 33 & 2.2 \\
\hline E4 & $\begin{array}{l}\text { I had to do things that put me at risk of infection (e.g., } \\
\text { commute to work, do my job, go shopping). }\end{array}$ & 438 & 29.2 & 578 & 38.5 & 357 & 23.8 & 127 & 8.5 \\
\hline E5 & $\begin{array}{l}\text { My workload increased quantitatively (e.g., increased } \\
\text { overtime). }\end{array}$ & 1258 & 83.9 & 85 & 5.7 & 112 & 7.5 & 45 & 3.0 \\
\hline E6 & $\begin{array}{l}\text { I worried about relationships at work (e.g., everyday } \\
\text { relationships, differences of opinion regarding } \\
\text { infection prevention). }\end{array}$ & 1097 & 73.1 & 148 & 9.9 & 162 & 10.8 & 93 & 6.2 \\
\hline E7 & $\begin{array}{l}\text { I began to experience mental health issues resulting } \\
\text { from the coronavirus outbreaks' effects. }\end{array}$ & 885 & 59.0 & 246 & 16.4 & 285 & 19.0 & 84 & 5.6 \\
\hline E8 & $\begin{array}{l}\text { Concerns about my relationship with my } \\
\text { boyfriend/girlfriend/a close friend (e.g., unable to get } \\
\text { in contact with them, not getting along, concerns } \\
\text { about infection). }\end{array}$ & 961 & 64.1 & 253 & 16.9 & 209 & 13.9 & 77 & 5.1 \\
\hline E9 & $\begin{array}{l}\text { My responsibilities at home increased, e.g., more } \\
\text { childcare, eldercare, housework. }\end{array}$ & 912 & 60.8 & 229 & 15.3 & 243 & 16.2 & 116 & 7.7 \\
\hline E10 & $\begin{array}{l}\text { Concerns about online relationships (hassles, } \\
\text { arguments, abuse). }\end{array}$ & 1352 & 90.1 & 61 & 4.1 & 66 & 4.4 & 21 & 1.4 \\
\hline E11 & Being unable to meet people. & 251 & 16.7 & 566 & 37.7 & 435 & 29.0 & 248 & 16.5 \\
\hline E12 & Uncertainty about the future. & 209 & 13.9 & 311 & 20.7 & 540 & 36.0 & 440 & 29.3 \\
\hline E13 & I slept less, and the quality of my sleep decreased. & 817 & 54.5 & 245 & 16.3 & 315 & 21.0 & 123 & 8.2 \\
\hline E14 & Insufficient exercise. & 326 & 21.7 & 631 & 42.1 & 381 & 25.4 & 162 & 10.8 \\
\hline E15 & $\begin{array}{l}\text { The time I spent social networking, surfing the web, } \\
\text { gaming, or shopping online increased. }\end{array}$ & 359 & 23.9 & 891 & 59.4 & 185 & 12.3 & 65 & 4.3 \\
\hline
\end{tabular}




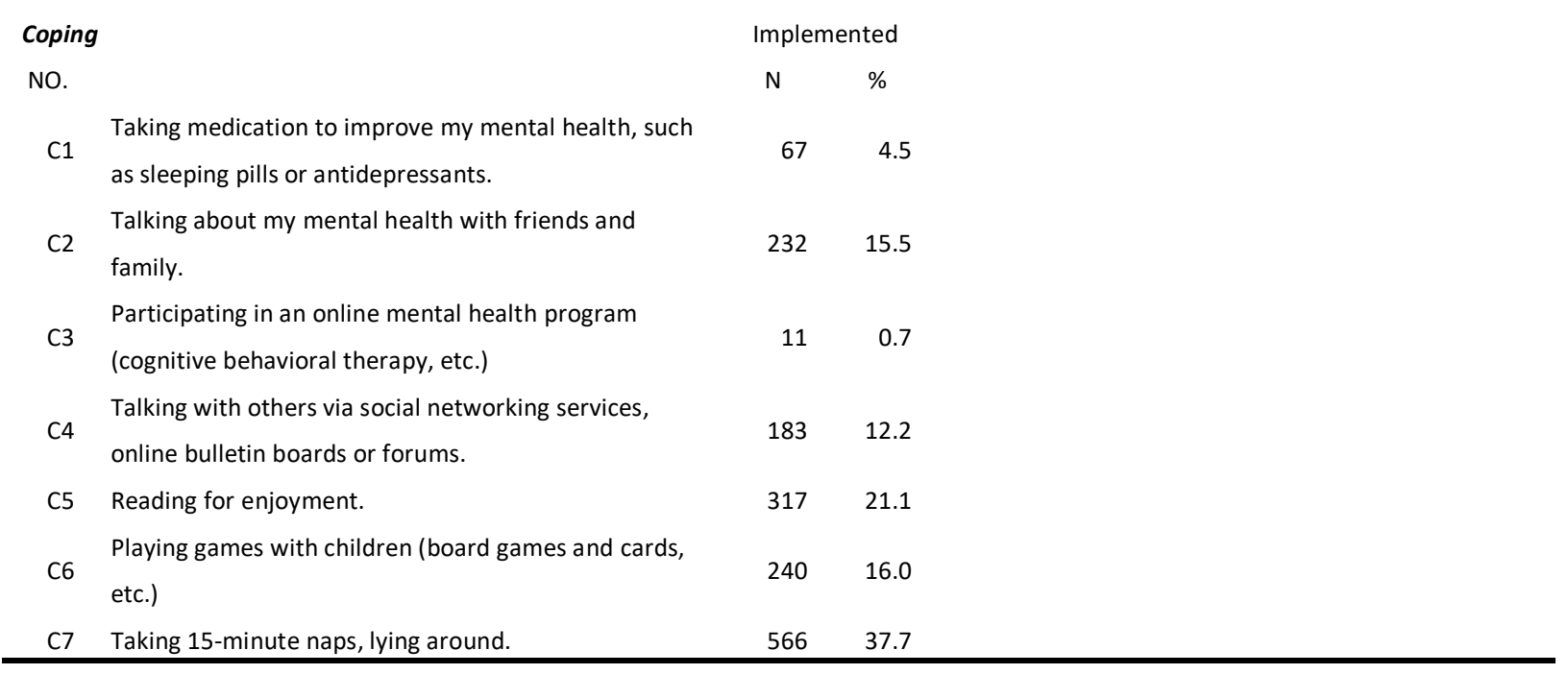

Note. $N=1500$.

\subsubsection{Psychological Scales}

The ten-item personality inventory [16] was used as a personality index. This scale measures an individual's personality based on the five-factor model (the Big Five). The Japanese version (TIPI-J) was developed, and its validity was confirmed by Oshio [17]. Participants' responses to two questions for each of the five factors were rated on a seven-point scale (Extraversion [EXT], Agreeableness [AGR], Conscientiousness [CON], Neuroticism [NEU], Openness [OPE]). Each factor was scored from 0 to 14 points.

A shortened version [18] of the Japanese edition of the Multidimensional Scale of Perceived Social Support [19], comprising of seven items with high factor loadings taken from the original scale, was used as an index of social support. The participants responded to items such as "my family lends a hand to support me mentally when I need it" rated on a seven-point scale. The total score ranged from 7 to 49 points.

The SF-8TM was used as an index of mental and physical health [20]. The Japanese version of the SF-8TM was developed by Fukuhara [21]. A standard physical component summary (PCS) and mental component summary (MCS) with a mean score of 50 and standard deviation (SD) of 10 were calculated based on an algorithm derived from the standard scores of 2,284 Japanese individuals [21]. A higher score indicated better health for both summary scores.

The Kessler (K6) scale was used as an index of psychological distress [22]. The Japanese version of the $K 6$ was developed by Furukawa [23]. Scores on the six-item scale range from 0 to 24 points. A higher score indicates higher psychological distress. The mean \pm SD from a community sample of $85,154,382$ Japanese individuals aged 15 or older was $3.34 \pm 3.39$ [24].

The Suicide Behaviors Questionnaire-Revised (SBQ) was used as an index of suicidal ideation [25]. This scale measures the level of suicidal ideation and comprises four questions, each assessed on a five-to-seven-point scale. The total score ranges from 3 to 18 points. This scale was translated into Japanese using back-translation. 


\subsection{Statistical Analysis}

All variables used in this analysis are enlisted in Table 2. Categorical variables were converted into binary. The following responses were entered as 1 while any other response was entered as 0. Sex: Female; the highest level of education: junior college graduate; comorbidity: psychiatric outpatient; employment status: full-time employed or unemployed (excluding student); housing facilities: garden or balcony; preschool-age children in the home: 3; having been infected with COVID-19: infection suspected; contact with infected persons: had to contact; the extent of voluntary restriction of outside activity: did not refrain from going out and did not practice social distancing. First, Spearman's rank correlation coefficient was calculated using IBM SPSS 26, and a two-tailed significance test was performed to explore correlations between variables. The significance level was $p<0.0001$. Subsequently, a mediation model was considered SEM using Mplus 8.4. A hypothetical model of the direct and indirect effects mediated by stressors of demographic characteristics, COVID-19-related elements, coping, personality, and social support was created on PCS, MCS, K6, and SBQ, respectively, as objective variables. Paths that did not reach the significance level $(p<0.0001)$ were temporarily removed after which, the paths deemed necessary based on $\chi 2$ value were reincorporated to obtain the optimal model. The standards for model fitting were standardized root mean squared residual (SRMR) $\leq 0.08$, the comparative fit index $(\mathrm{CFI}) \geq 0.95$, and the root mean square error of approximation (RMSEA) $\leq 0.06$ [26]. 
Table 2 Correlation between the variables.

\begin{tabular}{|c|c|c|c|c|c|c|c|c|c|c|c|c|c|c|c|c|c|c|c|c|c|c|c|}
\hline & & 1 & 2 & 3 & 4 & 5 & 6 & 7 & 8 & 9 & 10 & 11 & 12 & 13 & 14 & 15 & 16 & 17 & 18 & 19 & 20 & 21 & 22 \\
\hline 1 & PCS & 1 & & & & & & & & & & & & & & & & & & & & & \\
\hline 2 & MCS & -.16 & 1 & & & & & & & & & & & & & & & & & & & & \\
\hline 3 & K6 & -.12 & -.62 & 1 & & & & & & & & & & & & & & & & & & & \\
\hline 4 & SBQ & -.11 & $\overline{-.28}$ & .47 & 1 & & & & & & & & & & & & & & & & & & \\
\hline 5 & Extraversion & & & -.18 & -.23 & 1 & & & & & & & & & & & & & & & & & \\
\hline 6 & Agreeableness & & .13 & -.23 & -.21 & & 1 & & & & & & & & & & & & & & & & \\
\hline 7 & Conscientiousness & & .09 & -.16 & -.19 & .20 & .22 & 1 & & & & & & & & & & & & & & & \\
\hline 8 & Neuroticism & & -.34 & .47 & .34 & -.27 & -.28 & -.31 & 1 & & & & & & & & & & & & & & \\
\hline 9 & Openness & & & & & .35 & .15 & $\overline{.22}$ & -.22 & 1 & & & & & & & & & & & & & \\
\hline 10 & Social Support & .11 & .12 & -.29 & -.38 & .36 & .25 & .10 & -.18 & .14 & 1 & & & & & & & & & & & & \\
\hline 11 & Age & & .09 & & & & & .14 & & & -.20 & 1 & & & & & & & & & & & \\
\hline 12 & Gender: Female & & -.13 & & & & & & .18 & -.12 & .16 & -.14 & 1 & & & & & & & & & & \\
\hline 13 & Education: Junior college & & & & & & & & & & & & .20 & 1 & & & & & & & & & \\
\hline 14 & Psychiatric outpatient & -.11 & -.16 & .22 & .27 & -.15 & & & .19 & & -.12 & & & & 1 & & & & & & & & \\
\hline 15 & Employment: Full-time & & & & & .13 & & & -.13 & & & & -.31 & & & 1 & & & & & & & \\
\hline 16 & Employment: Unemployed & & & & & -.14 & & & & & -.13 & .09 & & & .13 & -.20 & 1 & & & & & & \\
\hline 17 & Workplace commutation & & & & & & & & -.14 & & & & -.26 & & & .53 & -.32 & 1 & & & & & \\
\hline 18 & Housing facilities: Garden & & & & & & & & & & & .14 & & & & $\overline{-.10}$ & & & 1 & & & & \\
\hline 19 & Number of preschool children & & & & & .10 & & & & & .17 & -.10 & .11 & & & & -.12 & -.18 & & 1 & & & \\
\hline 20 & Preschool children: Three & & & & & & & & & & & & & & & & & & & .21 & 1 & & \\
\hline 21 & COVID-19 infection: Suspected & & & .11 & .12 & & & & & & & & & & & & & & & & & 1 & \\
\hline 22 & Recent contact with patients: Yes & & & & & & & & & & & & & & & & & & & & & .12 & 1 \\
\hline 23 & Voluntary restriction: None & & & & & & & & & & & & & & & & & & & & & & \\
\hline 24 & E1 & & -.18 & .22 & .14 & & & & & & & & & & & & & & & & & & \\
\hline 25 & E2 & -.11 & & .11 & .12 & & & & & & -.12 & & & & & & & & & & & & .10 \\
\hline 26 & E3 & -.14 & -.13 & .24 & .20 & & -.12 & & .14 & & -.10 & & & & .19 & & & & & & .09 & & \\
\hline 27 & E4 & & -.22 & .26 & .19 & & -.10 & & .17 & & & & .12 & & & & -.09 & .15 & & & & & \\
\hline 28 & E5 & -.10 & -.09 & .11 & & & & & & & & & & & & .25 & -.12 & .30 & & & & & \\
\hline 29 & E6 & & -.18 & .23 & .14 & & -.10 & & & & -.12 & & & & & .29 & -.16 & .36 & & & & & \\
\hline 30 & E7 & -.14 & -.44 & .53 & .31 & & -.11 & & .28 & & -.16 & & .13 & & .17 & & & & & & & .09 & \\
\hline 31 & E8 & & $\overline{-.26}$ &.$\overline{25}$ & $\overline{.15}$ & & & & .11 & & & -.13 & .12 & & & & & & & & & & \\
\hline 32 & E9 & & -16 & .13 & & & & & & & & .15 & .17 & .09 & & -.09 & -.11 & -.12 & .10 & .47 & .10 & & \\
\hline 33 & E10 & -.14 & -.12 & .22 & .24 & & -.14 & & & & -.16 & -.09 & & & .13 & & & & & & & .11 & .10 \\
\hline 34 & E11 & & -.27 & .18 & & .22 & & & & & .27 & -.22 & .18 & & & & -.10 & -.10 & & .11 & & & \\
\hline 35 & E12 & & -.40 & .43 & .18 & & & & .26 & & & -.10 & .22 & & & -.12 & & -.10 & & & & & \\
\hline 36 & E13 & -.16 & -.37 &.$\overline{44}$ & .26 & & & -.09 & .23 & & -.14 & & & & .12 & & & & & & & & \\
\hline 37 & E14 & -.23 & $\frac{-.26}{-1}$ & .28 & .10 & & -.12 & & .12 & & & & & & & & & & & & & & \\
\hline 38 & E15 & & -.21 & .25 & .09 & & & & .11 & & & -.19 & .11 & & & & & & & & & & \\
\hline 39 & C1 & & -.16 & .20 & .21 & & & & .14 & & -.11 & & & & .49 & & & & & & & & \\
\hline 40 & C2 & & -.14 & .17 & .10 & & & & & & .16 & & .11 & & $\overline{.11}$ & -.13 & & -.11 & & & & & \\
\hline 41 & C3 & & & & & & & & & & & & & & & & & & & & & & \\
\hline 42 & C4 & & & & .13 & & & & & .09 & & -.13 & & & & & & & & & & & \\
\hline
\end{tabular}


OBM Integrative and Complementary Medicine 2021; 6(1), doi:10.21926/obm.icm.2101010

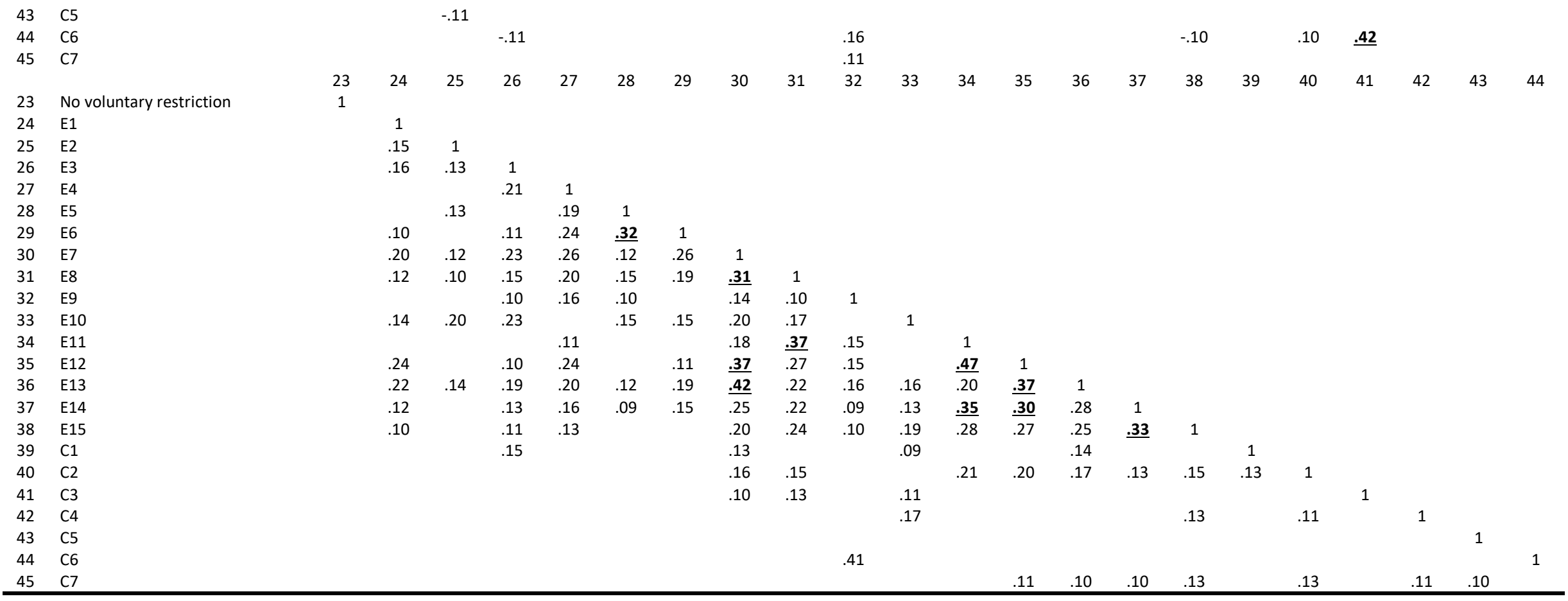

Note: Correlations significant only at $p<0.0001$ are shown. Correlations are in bold and underlined when $|\rho| \geq 0.30$. Abbreviations: PCS, physical component summary; MCS, mental component summary; SBQ, Suicide Behaviors Questionnaire-Revised Japanese version. 


\subsection{Ethics}

The participants voluntarily cooperated with the survey based on informed consent with written explanations. The research design was approved by the Research Ethics Review Committee of the Practical Psychology Institute, LLC (No. 2020001).

\section{Results}

A minimum of one response was collected for each of the 47 prefectures of Japan. The participant characteristics are shown in Tables 1, Table 3 and Table 4. According to Japan's February 2020 Labour Force Survey, 51.7\% of the population aged 15 years or older are female and $4.5 \%$ are self-employed/sole proprietors [27]. Compared to the Japanese workforce population, there were slightly more females and self-employed/sole proprietors among the participants in this study. Further, mean scores for MCS and K6 (43.43 and 6.49, respectively) were less than the standard scores in Japan under ordinary circumstances.

Table 2 shows the correlation coefficients between each variable. Several significant one-to-one relationships were observed between the variables.

Table 3 Characteristics of the study participants.

\begin{tabular}{|c|c|c|c|c|c|}
\hline & $\mathrm{N}$ & $\%$ & & $\mathrm{~N}$ & $\%$ \\
\hline Age & & & Employment before epidemic & & \\
\hline 18 or 19 & 23 & 1.5 & student & 75 & 5 \\
\hline $20-24$ & 138 & 9.2 & self-employed & 157 & 10.5 \\
\hline $25-29$ & 240 & 16 & company manager & 9 & 0.6 \\
\hline $30-34$ & 314 & 20.9 & full-time & 528 & 35.2 \\
\hline $35-39$ & 256 & 17.1 & part-time & 180 & 12 \\
\hline $40-44$ & 232 & 15.5 & temporary workers & 158 & 10.5 \\
\hline $45-49$ & 125 & 8.3 & housewives/husbands & 254 & 16.9 \\
\hline $50-54$ & 95 & 6.3 & leave of absence & 36 & 2.4 \\
\hline $55-59$ & 42 & 2.8 & unemployed & 103 & 6.9 \\
\hline $60-64$ & 20 & 1.3 & Annual household income & & \\
\hline $65-69$ & 10 & 0.7 & $<18600$ USD & 292 & 19.5 \\
\hline$\geq 70$ & 5 & 0.3 & 18600 USD to 37200 USD & 425 & 28.3 \\
\hline Sex & & & 37300 USD to 74500 USD & 598 & 39.9 \\
\hline female & 969 & 64.6 & 74600 USD to 111800 USD & 146 & 9.7 \\
\hline male & 524 & 34.9 & 111900 USD to 149000 USD & 27 & 1.8 \\
\hline other & 7 & 0.5 & $\geq 149100$ USD & 12 & 0.8 \\
\hline Education & & & Workplace commutation & & \\
\hline junior high school & 28 & 1.9 & unemployed & 445 & 29.7 \\
\hline high school & 330 & 22 & nearly none & 430 & 28.7 \\
\hline junior college & 355 & 23.7 & half of the week or less & 183 & 12.2 \\
\hline university & 718 & 47.9 & more than half of the week & 118 & 7.9 \\
\hline graduate degree & 69 & 4.6 & almost every day & 324 & 21.6 \\
\hline Marital status & & & Housing facilities and surrounding environment & & \\
\hline unmarried & 659 & 43.9 & garden or balcony & 1184 & 78.9 \\
\hline divorced/widowed & 64 & 4.3 & Number of preschool-age children & & \\
\hline married [separated] & 50 & 3.3 & 0 & 1181 & 78.7 \\
\hline living with partner & 727 & 48.5 & 1 & 221 & 14.7 \\
\hline Ethnicity & & & 2 & 87 & 5.8 \\
\hline Asian & 1489 & 99.3 & 3 & 11 & 0.7 \\
\hline Black & 1 & 0.1 & Respondents' own COVID-19 infection status & & \\
\hline multiracial & 3 & 0.2 & fully recovered & 2 & 0.1 \\
\hline White & 2 & 0.1 & undergoing treatment & 1 & 0.1 \\
\hline Han Chinese & 3 & 0.2 & suspected to be infected & 13 & 0.9 \\
\hline Middle Eastern/Arab & 1 & 0.1 & none & 1484 & 98.9 \\
\hline other & 1 & 0.1 & Recent contact with infected persons & & \\
\hline Comorbidity & & & none & 1392 & 92.8 \\
\hline primary illness & 152 & 10.1 & suspected of having contact & 70 & 4.7 \\
\hline psychiatric outpatient status & 119 & 7.9 & yes & 38 & 2.5 \\
\hline
\end{tabular}


OBM Integrative and Complementary Medicine 2021; 6(1), doi:10.21926/obm.icm.2101010

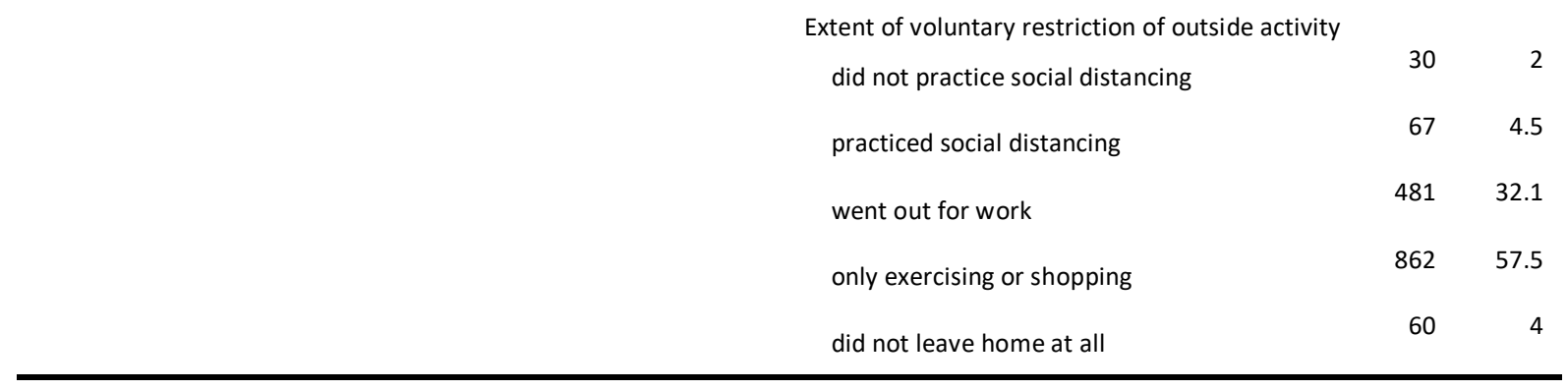

Note: $N=1500$

Table 4 Psychological measures: Descriptive statistics.

\begin{tabular}{lcccc}
\hline & Min & Max & Average & SD \\
\hline TIPI-J & 2 & 14 & 7.22 & 2.87 \\
Extraversion & 2 & 14 & 9.69 & 2.22 \\
Agreeableness & 2 & 14 & 7.18 & 2.61 \\
Conscientiousness & 2 & 14 & 8.79 & 2.67 \\
Neuroticism & 2 & 14 & 7.70 & 2.59 \\
Openness & & & & \\
Social Support & 7 & 49 & 35.18 & 9.19 \\
SF-8 & & & 51.04 & 6.70 \\
$\quad$ PCS & 19.91 & 68.27 & 43.43 & 8.14 \\
MCS & 13.69 & 62.30 & 6.49 & 5.34 \\
K6 & 0 & 24 & 5.79 & 2.99 \\
SBQ & 3 & 18 &
\end{tabular}

Note: $N=1500$. TIPI-J: Ten Item Personality Inventory Japanese version, PCS: physical component summary. MCS: mental component summary. SBQ: Suicide Behaviors Questionnaire-Revised Japanese version.

Tables 5 and 6 show the results of SEM. AGR (total/indirect $b=0.1$, confidence interval [Cl] [99.99\%]: 0.01 to 0.18 ), social support (total/indirect $b=0.03, \mathrm{Cl}: 0.01$ to 0.06 ), and psychiatric disorder (total $b=-2.69, \mathrm{Cl}:-5.32$ to -0.07 ; indirect $b=-0.3, \mathrm{Cl}:-0.78$ to 0.19 ns; direct $b=-0.14$, $\mathrm{Cl}:-0.39$ to $0.11 \mathrm{~ns})$ had significant total effects on PCS.

Table 5 Unstandardized path coefficients (b) estimated using structural equation modeling.

\begin{tabular}{|c|c|c|c|c|c|c|c|c|c|c|c|c|c|c|c|c|c|c|c|c|}
\hline & \multicolumn{2}{|c|}{ E1 } & \multicolumn{2}{|c|}{ E2 } & \multicolumn{2}{|c|}{ E3 } & \multicolumn{2}{|c|}{ E4 } & \multicolumn{2}{|c|}{ E5 } & \multicolumn{2}{|c|}{ E6 } & \multicolumn{2}{|c|}{ E7 } & \multicolumn{2}{|c|}{$\mathrm{E} 8$} & \multicolumn{2}{|c|}{ E9 } & \multicolumn{2}{|c|}{ E10 } \\
\hline & $b$ & $\mathrm{SE}$ & $\mathrm{b}$ & SE & b & SE & $b$ & $\mathrm{SE}$ & $\mathrm{b}$ & $\mathrm{SE}$ & $\mathrm{b}$ & $\mathrm{SE}$ & $\mathrm{b}$ & $\mathrm{SE}$ & $b$ & $\mathrm{SE}$ & $\mathrm{b}$ & $\mathrm{SE}$ & $\mathrm{b}$ & SE \\
\hline Extraversion & 0.04 & 0.01 & - & - & - & - & - & - & - & - & - & - & - & - & 0.03 & 0.01 & - & - & - & - \\
\hline Agreeableness & - & - & - & - & -0.02 & 0.01 & - & - & - & - & -0.02 & 0.01 & - & - & - & - & - & - & -0.03 & 0.01 \\
\hline Conscientiousness & - & - & . & - & - & - & - & - & - & . & - & - & - & . & - & - & - & - & - & - \\
\hline Neuroticism & 0.03 & 0.01 & - & - & 0.02 & 0.01 & 0.06 & 0.01 & - & - & 0.03 & 0.01 & 0.08 & 0.01 & 0.04 & 0.01 & 0.03 & 0.01 & - & - \\
\hline Openness & - & - & - & - & - & - & - & - & - & - & - & - & - & - & - & - & - & - & - & - \\
\hline Social Support & -0.01 & 0 & -0.01 & 0 & -0.01 & 0 & - & - & -0.01 & 0 & -0.01 & 0 & -0.01 & 0 & - & - & - & - & -0.01 & 0 \\
\hline Age & - & - & - & - & 0.02 & 0.01 & - & - & - & - & - & - & - & - & -0.05 & 0.01 & 0.09 & 0.01 & -0.02 & 0.01 \\
\hline Female & - & - & - & - & - & - & 0.25 & 0.05 & 0.12 & 0.04 & 0.16 & 0.05 & 0.21 & 0.04 & 0.14 & 0.04 & 0.29 & 0.04 & - & - \\
\hline Junior college & 0.15 & 0.06 & - & - & - & - & - & - & - & - & - & - & - & - & - & - & - & - & - & - \\
\hline Psychiatric outpatient & - & - & - & - & 0.31 & 0.10 & - & - & - & - & - & - & 0.38 & 0.09 & 0.19 & 0.09 & - & - & 0.20 & 0.07 \\
\hline Full-time & -0.14 & 0.05 & - & - & - & - & - & - & 0.23 & 0.05 & 0.32 & 0.06 & - & - & - & - & - & - & - & - \\
\hline Unemployed & - & - & - & - & - & - & - & - & - & - & -0.15 & 0.05 & - & - & - & - & -0.24 & 0.09 & - & - \\
\hline Commutation & - & - & - & - & -0.03 & 0.01 & 0.14 & 0.02 & 0.11 & 0.02 & 0.15 & 0.02 & - & - & - & - & -0.03 & 0.02 & - & - \\
\hline Garden & -0.16 & 0.06 & - & - & - & - & - & - & - & - & - & - & - & - & - & - & - & - & - & - \\
\hline $\mathrm{N}$ of Preschool children & -0.11 & 0.04 & 0.05 & 0.02 & - & - & - & - & 0.08 & 0.03 & - & - & - & - & - & - & 0.60 & 0.05 & - & - \\
\hline Three preschool children & 0.90 & 0.32 & & - & 0.55 & 0.29 & - & - & & - & - & - & - & - & - & - & -0.65 & 0.29 & - & - \\
\hline
\end{tabular}




\begin{tabular}{|c|c|c|c|c|c|c|c|c|c|c|c|c|c|c|c|c|c|c|c|c|}
\hline Suspected COVID-19 infection & 0.45 & 0.24 & 0.32 & 0.22 & - & - & - & - & - & - & - & - & 0.47 & 0.19 & - & - & - & - & 0.34 & 021 \\
\hline Recent contact with patients & - & - & 0.23 & 0.10 & 0.25 & 0.13 & - & - & - & - & - & - & - & - & - & - & - & - & 0.23 & 011 \\
\hline No voluntary restriction & - & - & - & - & - & - & - & - & - & - & - & - & - & - & - & - & - & - & - & - \\
\hline C1 & - & - & - & - & 0.21 & 0.14 & - & - & - & - & - & - & - & - & - & - & - & - & - & - \\
\hline C2 & 0.23 & 0.08 & - & - & 0.13 & 0.06 & 0.19 & 0.07 & 0.11 & 0.05 & 0.20 & 0.06 & 0.34 & 0.07 & 0.28 & 0.07 & - & - & - & - \\
\hline с3 & 0.64 & 0.31 & - & - & - & - & - & - & - & - & - & - & 0.92 & 0.21 & 1.12 & 0.23 & 0.69 & 0.23 & 0.70 & 031 \\
\hline C4 & - & - & - & - & - & - & - & - & - & - & - & - & - & - & - & - & - & - & 0.27 & 0.06 \\
\hline C5 & - & - & - & - & - & - & - & - & - & - & - & - & - & - & - & - & - & - & - & - \\
\hline C6 & - & - & - & - & - & - & - & - & - & - & - & - & - & - & - & - & 0.58 & 0.07 & - & - \\
\hline \multirow[t]{3}{*}{$\mathrm{C7}$} & - & - & - & - & - & - & - & - & - & - & - & - & - & - & - & - & - & - & 0.06 & 0.08 \\
\hline & \multicolumn{2}{|c|}{ E11 } & \multicolumn{2}{|c|}{ E12 } & \multicolumn{2}{|c|}{ E13 } & \multicolumn{2}{|c|}{ E14 } & \multicolumn{2}{|c|}{ E15 } & \multicolumn{2}{|c|}{ PCS } & \multicolumn{2}{|c|}{ MCS } & \multicolumn{2}{|c|}{ K6 } & \multicolumn{2}{|c|}{ SBQ } & & \\
\hline & b & SE & b & SE & b & SE & b & SE & b & SE & b & SE & b & SE & b & SE & b & SE & & \\
\hline Extraversion & 0.05 & 0.01 & 0.03 & 0.01 & - & - & 0.02 & 0.01 & 0.01 & 0.01 & - & - & - & - & -0.07 & 0.03 & -0.05 & 0.02 & & \\
\hline Agreeableness & - & - & - & - & - & - & -0.04 & 0.01 & - & - & - & - & - & - & -0.16 & 0.05 & - & - & & \\
\hline Conscientiousness & - & - & - & - & - & - & - & - & - & - & - & - & - & - & - & - & -0.09 & 0.03 & & \\
\hline Neuroticism & 0.04 & 0.01 & 0.09 & 0.01 & 0.08 & 0.01 & 0.04 & 0.01 & 0.03 & 0.01 & 0.18 & 0.07 & -0.5 & 0.07 & 0.44 & 0.04 & 0.14 & 0.03 & & \\
\hline Openness & - & - & - & - & 0.02 & 0.01 & - & - & - & - & - & - & -0.12 & 0.06 & - & - & 0.05 & 0.03 & & \\
\hline Social Support & 0.02 & 0 & - & - & -0.02 & 0 & - & - & - & - & - & - & 0.07 & 0.02 & -0.07 & 0.01 & -0.09 & 0.01 & & \\
\hline Age & -0.06 & 0.01 & - & - & - & - & -0.02 & 0.01 & -0.05 & 0.01 & -0.16 & 0.08 & - & - & - & - & - & - & & \\
\hline Female & 0.18 & 0.05 & 0.29 & 0.05 & - & - & - & - & - & - & - & - & - & - & - & - & 0.33 & 0.13 & & \\
\hline Junior college & - & - & - & - & - & - & - & - & - & - & - & - & - & - & - & - & - & - & & \\
\hline Psychiatric outpatient & - & - & - & - & - & - & - & - & - & - & -2.40 & 0.67 & - & - & 1.16 & 0.46 & 1.81 & 0.37 & & \\
\hline Full-time & - & - & - & - & - & - & - & - & - & - & - & - & - & - & - & - & - & - & & \\
\hline Unemployed & - & - & - & - & - & - & - & - & - & - & - & - & - & - & 0.79 & 0.35 & - & - & & \\
\hline Commutation & - & - & - & - & - & - & - & - & - & - & 0.31 & 0.10 & - & - & - & - & - & - & & \\
\hline Garden & - & - & - & - & - & - & - & - & - & - & 0.96 & 0.40 & 0.80 & 0.42 & -0.82 & 0.23 & - & - & & \\
\hline $\mathrm{N}$ of Preschool children & 0.08 & 0.03 & 0.12 & 0.03 & 0.17 & 0.04 & - & - & - & - & - & - & -0.56 & 0.29 & - & - & - & - & & \\
\hline Three preschool children & - & - & - & - & - & - & - & - & - & - & - & - & - & - & - & - & 1.59 & 0.74 & & \\
\hline Suspected COVID-19 infection & - & - & - & - & - & - & - & - & - & - & -5.57 & 2.24 & - & - & 3.70 & 1.30 & 2.41 & 0.49 & & \\
\hline Recent contact with patients & - & - & - & - & - & - & - & - & - & - & - & - & - & - & -1.70 & 0.58 & - & - & & \\
\hline No voluntary restriction & - & - & - & - & - & - & - & - & - & - & - & - & - & - & 2.12 & 0.88 & 0.91 & 0.53 & & \\
\hline $\mathrm{C} 1$ & - & - & - & - & 0.37 & 0.13 & - & - & - & - & - & - & -3.58 & 0.95 & 1.85 & 0.64 & 0.94 & 0.50 & & \\
\hline C2 & 0.37 & 0.06 & 0.4 & 0.06 & 0.43 & 0.07 & 0.26 & 0.06 & 0.20 & 0.05 & - & - & - & - & 0.48 & 0.25 & 0.33 & 0.18 & & \\
\hline с3 & 0.61 & 0.29 & 0.57 & 0.23 & - & - & 0.32 & 0.27 & - & - & - & - & - & - & 2.47 & 1.17 & - & - & & \\
\hline C4 & - & - & - & - & - & - & - & - & 0.18 & 0.05 & - & - & - & - & - & - & 0.71 & 0.20 & & \\
\hline C5 & - & - & - & - & - & - & - & - & - & - & - & - & - & - & -0.79 & 0.19 & - & - & & \\
\hline c6 & - & - & - & - & -0.14 & 0.06 & - & - & - & - & - & - & - & - & - & - & - & - & & \\
\hline C7 & - & - & 0.12 & 0.04 & 0.16 & 0.05 & 0.13 & 0.04 & 0.13 & 0.04 & 0.84 & 0.31 & - & - & - & - & - & - & & \\
\hline E1 & - & - & - & - & - & - & - & - & - & - & - & - & -0.5 & 0.19 & 0.32 & 0.11 & 0.12 & 0.07 & & \\
\hline E2 & - & - & - & - & - & - & - & - & - & - & -0.52 & 0.28 & - & - & - & - & 0.46 & 0.14 & & \\
\hline E3 & - & - & - & - & - & - & - & - & - & - & -0.55 & 0.25 & - & - & 0.34 & 0.13 & 0.23 & 0.11 & & \\
\hline E4 & - & - & - & - & - & - & - & - & - & - & - & - & - & - & - & - & - & - & & \\
\hline E5 & - & - & - & - & - & - & - & - & - & - & -0.60 & 0.25 & -0.44 & 0.24 & - & - & - & - & & \\
\hline E6 & - & - & - & - & - & - & - & - & - & - & - & - & - & - & 0.21 & 0.11 & - & - & & \\
\hline E7 & - & - & - & - & - & - & - & - & - & - & - & - & -2.12 & 0.23 & 1.30 & 0.14 & 0.26 & 0.09 & & \\
\hline E8 & - & - & - & - & - & - & - & - & - & - & - & - & - & - & - & - & 0.16 & 0.08 & & \\
\hline E9 & - & - & - & - & - & - & - & - & - & - & - & - & -0.30 & 0.17 & - & - & -0.21 & 0.06 & & \\
\hline E10 & - & - & - & - & - & - & - & - & - & - & -0.64 & 0.34 & - & - & 0.63 & 0.20 & 0.46 & 0.14 & & \\
\hline E11 & - & - & - & - & - & - & - & - & - & - & 0.53 & 0.22 & -0.94 & 0.21 & - & - & - & - & & \\
\hline E12 & - & - & - & - & - & - & - & - & - & - & 0.38 & 0.19 & -1.04 & 0.21 & 0.75 & 0.12 & 0.17 & 0.07 & & \\
\hline E13 & - & - & - & - & - & - & - & - & - & - & -0.67 & 0.19 & -0.76 & 0.22 & 0.60 & 0.12 & 0.14 & 0.08 & & \\
\hline E14 & - & - & - & - & - & - & - & - & - & - & -1.77 & 0.21 & -0.41 & 0.22 & 0.32 & 0.12 & - & & & \\
\hline E15 & - & - & - & - & - & - & - & - & - & - & - & - & - & - & 0.32 & 0.14 & -0.15 & 0.10 & & \\
\hline
\end{tabular}

Note: Paths removed from the model are marked with a "-". Model fit indices: $\chi 2(605)=638.48$, $\mathrm{p}=0.168, \mathrm{CFI}=.996, \mathrm{RMSEA}=.006, \mathrm{SRMR}=.025$.

Table 6 Standardized total effects $(\beta)$ estimated using the structural equation modeling.

\begin{tabular}{|c|c|c|c|c|c|c|c|c|c|c|c|c|c|c|c|c|c|c|c|c|}
\hline & E1 & & E2 & & E3 & & E4 & & E5 & & E6 & & E7 & & E8 & & E9 & & E10 & \\
\hline & $\beta$ & SE & $\beta$ & SE & $\beta$ & SE & $\beta$ & SE & $\beta$ & SE & $\beta$ & SE & $\beta$ & SE & $\beta$ & SE & $\beta$ & SE & $\beta$ & SE \\
\hline Extraversion & & & - & - & - & - & - & - & - & - & - & - & - & - & & & - & - & - & - \\
\hline Agreeableness & - & - & - & - & & & - & - & - & - & & & - & - & - & - & - & - & & \\
\hline Conscientiousness & - & - & - & - & - & - & - & - & - & - & - & - & - & - & - & - & - & - & - & - \\
\hline Neuroticism & & & - & - & & & 0.18 & 0.02 & - & - & & & 0.23 & 0.02 & & & & & - & - \\
\hline Openness & - & - & - & - & - & - & - & - & - & - & - & - & - & - & - & - & - & - & - & - \\
\hline Social Support & & & & & & & - & - & & & & & -0.13 & 0.02 & - & - & - & - & -0.14 & 0.03 \\
\hline Age & - & - & - & - & & & - & - & - & - & - & - & - & - & -0.11 & 0.02 & 0.18 & 0.02 & & \\
\hline Female & - & - & - & - & - & - & 0.13 & 0.02 & & & & & 0.11 & 0.02 & & & 0.14 & 0.02 & - & - \\
\hline Junior college & & & - & - & - & - & - & - & - & - & - & - & - & - & - & - & - & - & - & - \\
\hline Psychiatric outpatient & - & - & - & - & & & - & - & - & - & - & - & 0.11 & 0.03 & & & - & - & & \\
\hline Full-time & & & - & - & - & - & - & - & 0.15 & 0.03 & 0.17 & 0.03 & - & - & - & - & - & - & - & - \\
\hline Unemployed & - & - & - & - & - & - & - & - & - & - & & & - & - & - & - & & & - & - \\
\hline Commutation & - & - & - & - & & & 0.22 & 0.02 & 0.23 & 0.03 & 0.26 & 0.03 & - & - & - & - & & & - & - \\
\hline Garden & & & - & - & - & - & - & - & - & - & - & - & - & - & - & - & - & - & - & - \\
\hline$N$ of Preschool children & & & & & - & - & - & - & & & - & - & - & - & - & - & 0.36 & 0.03 & - & - \\
\hline Three preschool children & & & - & - & & & - & - & - & - & - & - & - & - & - & - & & & - & - \\
\hline Suspected COVID-19 infection & & & & & - & - & - & - & - & - & - & - & & & - & - & - & - & & \\
\hline Recent contact with patients & - & - & & & & & - & - & - & - & - & - & - & - & - & - & - & - & & \\
\hline No voluntary restriction & - & - & - & - & - & - & - & - & - & - & - & - & - & - & - & - & - & - & - & - \\
\hline C1 & - & - & - & - & & & - & - & - & - & - & - & - & - & - & - & - & - & - & - \\
\hline C2 & & & - & - & & & & & & & & & 0.13 & 0.03 & 0.11 & 0.03 & - & - & - & - \\
\hline с3 & & & - & - & - & - & - & - & - & - & - & - & & & & & & & & \\
\hline
\end{tabular}




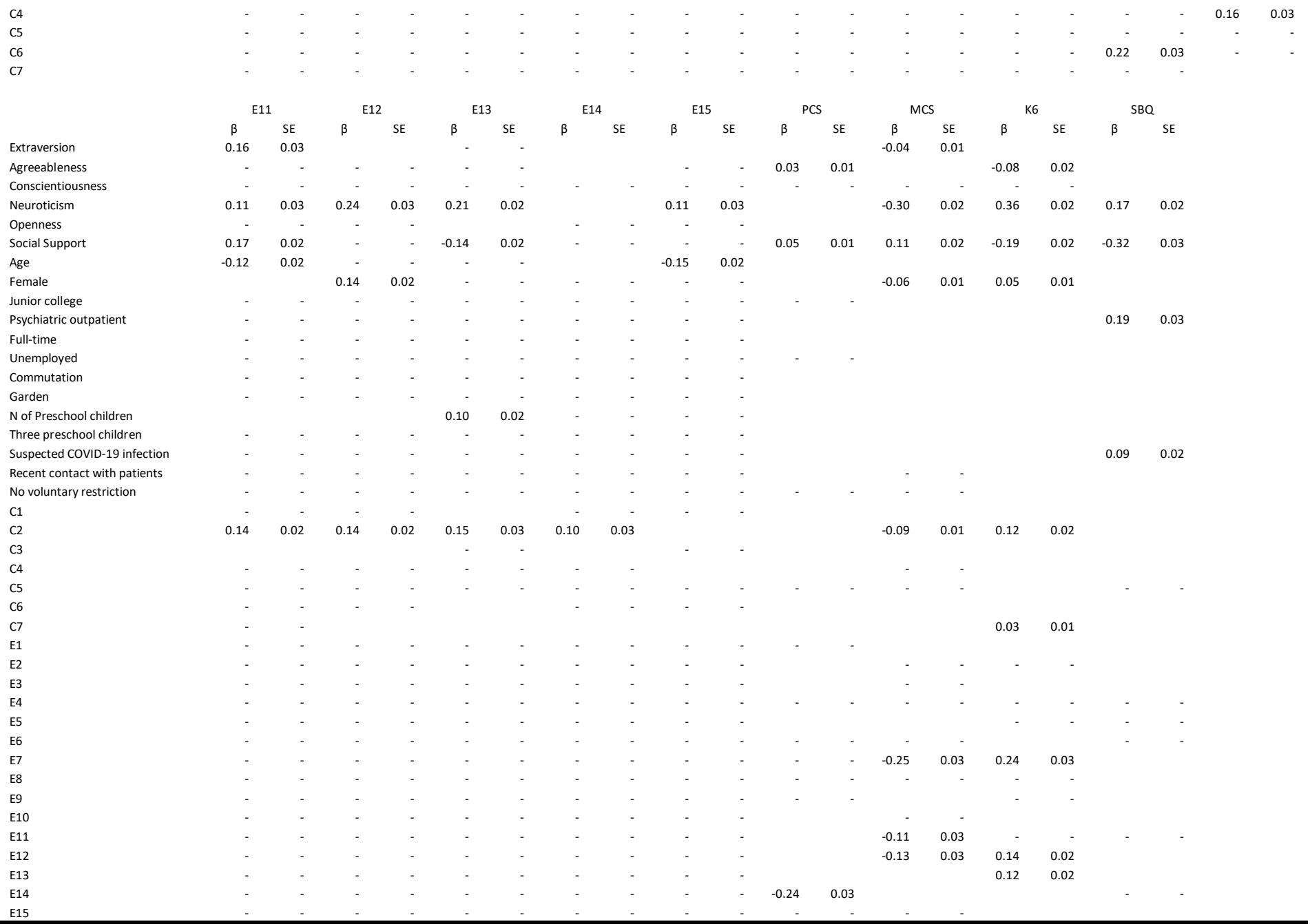

Note: Paths removed from the model are marked with a "-". Only coefficients significant at $p<$ 0.0001 are shown. Model fit indices: $\chi 2(605)=638.48, p=0.168, C F I=.996$, RMSEA $=.006$, SRMR $=.025$.

EXT (total/indirect $b=-0.1, \mathrm{Cl}:-0.18$ to -0.03 ), NEU (total $b=-0.9, \mathrm{Cl}:-1.19$ to -0.61 ; indirect $b$ $=-0.4, \mathrm{Cl}:-0.55$ to -0.25 ), social support (total $b=0.1, \mathrm{Cl}: 0.02$ to 0.18 ; indirect $b=0.03, \mathrm{Cl}: 0$ to 0.07 ), being female (total/indirect $b=-1.05, \mathrm{Cl}:-1.64$ to -0.45 ), psychiatric disorder (total/indirect $b=-0.82, \mathrm{Cl}:-1.61$ to -0.03 ), $\mathrm{C} 1$ (total $b=-3.86, \mathrm{Cl}:-7.58$ to -0.14 ; indirect $b=-0.28, \mathrm{Cl}:-0.79$ to 0.22 ns.; direct $b=-3.58, \mathrm{Cl}:-7.28$ to $0.12 \mathrm{~ns}$ ), $\mathrm{C} 5$ (total/indirect $b=-2.09, \mathrm{Cl}:-3.16$ to -1.01 ), and C7 (total/indirect $b=-3.76, \mathrm{Cl}:-6.9$ to -0.62 ) had significant total effects on MCS.

AGR (total $b=-0.19, \mathrm{Cl}:-0.37$ to -0.02 ; indirect $b=-0.04, \mathrm{Cl}:-0.08$ to 0 ), NEU (total $b=0.7, \mathrm{Cl}$ : 0.52 to 0.88 ; indirect $b=0.26, \mathrm{Cl}: 0.17$ to 0.36 ), social support (total $b=-0.11, \mathrm{Cl}:-0.16$ to -0.06 ; indirect $b=-0.04, \mathrm{Cl}:-0.06$ to -0.02 ), being female (total/indirect $b=0.52, \mathrm{Cl}: 0.2$ to 0.84 ), psychiatric disorder (total $b=1.89, \mathrm{Cl}: 0.05$ to 3.73 ; indirect $b=0.73, \mathrm{Cl}: 0.11$ to 1.35 ), $\mathrm{C} 5$ (total $b=$ 1.78, Cl: 0.69 to 2.88; indirect $b=1.3, \mathrm{Cl}: 0.62$ to 1.98 ), C7 (total $b=4.84,99.99 \% \mathrm{Cl}$ : 0.36 to 9.31; indirect $b=2.37, \mathrm{Cl}: 0.68$ to 4.05 ), $\mathrm{C} 16$ (total/direct $b=-0.79, \mathrm{Cl}:-1.55$ to -0.04 ), and $\mathrm{C} 30$ had significant total effects on $\mathrm{K} 6$.

$\mathrm{NEU}$ (total $b=0.19, \mathrm{Cl}: 0.08$ to 0.29 ; indirect $b=0.05, \mathrm{Cl}: 0.01$ to 0.09 ), social support (total $b=-$ $0.1, \mathrm{Cl}:-0.14$ to -0.07 ; indirect $b=-0.01, \mathrm{Cl}:-0.03$ to 0 ns.; direct $b=-0.09, \mathrm{Cl}:-0.12$ to -0.05 ), psychiatric disorder (total $b=2.1, \mathrm{Cl}: 0.67$ to 3.53 ; indirect $b=0.29, \mathrm{Cl}:-0.02$ to 0.6 ns.; $\operatorname{direct} b=$ 
$1.81, \mathrm{Cl}: 0.37$ to 3.25 ), suspected COVID-19 infection (total $b=2.89, \mathrm{Cl}: 0.77$ to 5.01 ; indirect $b=$ $0.48, \mathrm{Cl}:-0.28$ to 1.24 ns.; direct $b=2.41, \mathrm{Cl}: 0.51$ to 4.32 ), and $\mathrm{C} 8$ (total $b=0.8, \mathrm{Cl}: 0.03$ to 1.58 ; indirect $b=0.1, \mathrm{Cl}:-0.09$ to $0.28 \mathrm{~ns}$; $b=0.71, \mathrm{Cl}:-0.08$ to $1.49 \mathrm{~ns}$.) had significant total effects on SBQ.

Figure 1 shows a path diagram of the main part of the SEM results. The stressors with a significant impact on the four outcome measures were E7, E11, E12, E13, and E14. Three of the personality subscales (extraversion, agreeableness, and neuroticism); social support; age; sex (female); psychiatric hospitalization; garden residence; the number of infants; suspected COVID-19 infection; and copings (except C6) had significant indirect and/or direct effects on the four outcome measures.

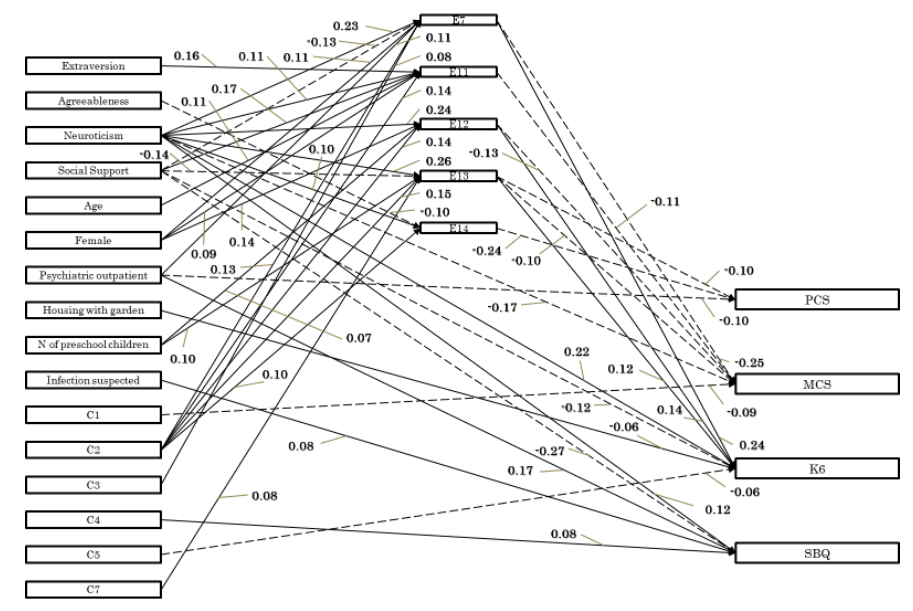

Figure 1 Standardized direct effects $(\beta)$ estimated using structural equation modeling. Model fit indices: $\chi 2(605)=638.48, p=0.168, C F I=.996$, RMSEA $=.006$, SRMR $=.025$. Only variables and paths that directly and indirectly affected the four outcome measures are shown. The unstandardized coefficients are illustrated in Table 5 for direct influence relationships for all variables. Abbreviations: PCS, physical component summary; MCS, mental component summary; SBQ, Suicide Behaviors Questionnaire-Revised Japanese version; C1, Taking medication; $\mathrm{C} 2$, Talking about mental health with friends and family; C3, Participating in an online program; C4, Talking with others via social networking services and so on; C5, Reading for enjoyment; C7, Taking 15-minute naps; E7, Experiencing mental health issues; E11, Being unable to meet people; E12, Uncertainty about the future; E13, Sleep problems; E14, Insufficient exercise.

\section{Discussion}

This study investigated the effects of demographic characteristics, elements related to SARSCoV-2, coping, personality, and social support on physical and mental health, psychological distress, and suicidal ideation in Japan immediately after the rescinding of the 7 weeks of restrictions on outings, inter-prefectural travel, and business activities, which begun on April 7, 2020, due to the state of emergency declaration. The results identified several direct and indirect relationships presumed to be due to the influence of the outbreak.

Physical health was found to have a positive relationship with AGR and social support, and a negative relationship with a psychiatric disorder. The effects of AGR and social support were completely mediated by stressors. AGR was suggested to promote physical health by weakening 
"difficulty in receiving medical care for a chronic disease," "problems with interpersonal relationships online," and "insufficient exercise," while social support did the same by weakening "problems with interpersonal relationships online," "reduction in sleep time and quality," and through "being unable to meet people,", respectively.

Mental health was found to have a positive relationship with social support, and a negative relationship with EXT, NEU, being female, psychiatric disorder, "using psychotropic drugs," "talking about one's mental health," and "participating in remote programs." The effects of social support and NEU were partially mediated by stressors, while the effects of EXT, being female, psychiatric disorder, "talking about one's mental health," and "participating in remote programs" were completely mediated. The mental health was most impacted by "mental health issues related to COVID-19." This factor was in turn influenced by NEU, social support, being a junior college graduate, and so forth, psychiatric disorder, and "talking about one's mental health." Increasing awareness in people about these characteristics and offering social support may be useful in promoting mental health.

Psychological distress was found to have a negative relationship with AGR, social support, and "reading for pleasure," and a positive relationship with NEU, being female, "talking about one's mental health," "participating in remote programs," and "15-min naps." The effects of NEU, psychiatric disorder, "talking about one's mental health," and "participating in remote programs" were partially mediated, while the effects of being female and "15-min naps" were completely mediated by stressors. NEU and "mental health issues related to COVID-19" had relatively large impacts on psychological distress. The effects of NEU were partially mediated by "large reduction in household income," "difficulty in receiving medical care for a chronic disease," "problems with interpersonal relationships in the workplace," "mental health issues related to COVID-19," "uncertainty about the future," "reduction in sleep time and quality," "insufficient exercise," and "increased time online." People with high NEU were more likely to perceive life events accompanying the SARS-CoV-2 outbreak as stressors. "Mental health issues related to COVID-19" were impacted by NEU, social support, being female, psychiatric disorder, and "talking about one's mental health." Calling attention to people with high NEU and offering support to people experiencing these stressors may help to alleviate psychological distress.

Suicidal ideation was found to have a negative relationship with social support, and a positive relationship with NEU, psychiatric disorder, suspected infection with SARS-CoV-2, and "talking with others online." Although the total effects of the stressors "increased responsibilities at home" and "increased time online" were not significant, each of them was shown to reduce suicidal ideation in the optimal model (Table 5). Although neither of them is generally considered factors that promote health, they likely worked to lessen suicidal ideation by preserving connections with others.

A positive total effect was found for "talking with others online." The positive effect through the mediator "concerns about online relationships" and the negative effect through "increased time online" coupled with the positive direct effect was surmised to ultimately increase suicidal ideation.

Social support had the greatest effect on suicidal ideation and was concluded to directly reduce suicidal ideation as no mediating effects were observed. Loss of connection is in some perspectives seen as a vital factor facilitating suicidal ideation [28]. In contrast, experiencing social support signifies a connection between the self and others and social support in this way may reduce suicidal ideation. 
Although significant correlations between the independent variables and objective variables were identified, the extent of the correlations did not undermine the validity of conducting SEM, including all variables to the model independently. From the comparison between Table 2 with Table 6 and Figure 1, the influence of many independent variables was smaller in the SEM than the one-to-one correlation coefficient. This suggests that the effect of a particular independent variable on an outcome can be predicted more accurately by considering the effects of other variables. For example, the correlation between Neuroticism and SBQ was $r=0.34$ (Table 2), while the overall effect of neuroticism on SBQ was $\beta=0.17$ (Table 6). In contrast, the relationship between social support and SBQ was $r=-0.38$ and $\beta=-0.32$. Looking at the correlation coefficients alone, neuroticism and social support appear to have the same strength of relationship to suicidal ideation; however, assuming the overall relationship of other variables, the effect of social support is observed to be greater. Similarly, although some copings showed undesirable correlations with outcome measures (negative correlation with MCS, positive correlation with K6 and SBQ), these effects appeared to be quite small (C2 to MCS, $\beta=-0.09$; $C 2$ to K6, $\beta=0.19 ; C 7$ to K6, $\beta=0.03$ ).

This study revealed novel findings of social support. Namely, the effects of social support were compared to other factors, and factors mediating the effects of social support were clarified. The present results suggest that social support has a particularly marked effect on reducing suicidal ideation. Further, the results suggest that the effects of social support impact mental and physical health and psychological distress by reducing the stressors accompanying "problems with interpersonal relationships online," "reduction in sleep time and quality," "reduction in household income," and "mental health issues related to COVID-19," as well as increasing the stressors accompanying "being unable to meet people."

This study has provided many insights into the effects of the SARS-CoV-2 outbreak on the mental and physical health of the general public. However, some limitations must be considered. First is the limited number of participants. Responses were collected from all over Japan, and looking at the participants' attributes, sex, and employment status does not reveal any evident deviation from the attributes of the country as a whole. However, as data were collected from 1,500 patients to represent all persons aged 18 and older in Japan, with a population of approximately 108 million, and as participants were recruited through an online outsourcing service, we must be careful in deciding that the results of this study represent the characteristics of all Japanese. Also, as a crosssectional study design was applied, the results cannot be used to confirm causal relationships. For example, coping not only influences stressors but experiencing stressors also results in coping. Thus, the order of these relationships should be considered. Longitudinal research or analysis of adjusted effects could deepen our understanding of this point. Moreover, the distinction between a stressor and perceived stress was not clear in this study. A detailed study is required to distinguish between each of them, the stressor purely as an event and the perceived stress, which represents the degree to which the stressor is perceived, and how coping is involved in the degree and relationship between these two. Finally, the present study does not adequately examine the biological aspects behind the stressor or perceived stress-causing serious mental health problems such as psychological distress and suicidal ideation. Measuring in vivo information such as serum 25Hydroxyvitamin D levels will bring us closer to uncovering the mechanisms of psychosocialbiological human responses under spreading COVID-19 infection. 


\section{Conclusions}

This study investigated the complex relationships of demographic characteristics, factors related to SARS-CoV-2, personality, social support, coping, and stressors with mental and physical health in the Japanese immediately after the request for voluntary restrictions of outings and business activities due to the SARS-CoV-2 outbreak was rescinded. The strength of the impact of each factor differed for physical health, mental health, psychological distress, and suicidal ideation. However, in all cases, the results suggested that some attributes such as sex and personality, and some elements related to infection such as suspected infection with SARS-CoV-2 had direct effects on physical and mental health and that there may also be effects mediated by stressors such as "insufficient exercise" or "mental health issues related to COVID-19." As the effects of the SARSCoV-2 pandemic continue globally, it is essential to prioritize resources and provide support to those at particularly high risk and offer effective evidence-based psychosocial interventions.

\section{Author Contributions}

Keita Kiuchi contributed to conceptualization, methodology, formal analysis, investigation, writing the draft, visualization and project administration. Katsumasa Kishi contributed to conceptualization, methodology, validation, and reviewing and editing the draft. Kanto Araki contributed to conceptualization, methodology, validation, and reviewing and editing the draft.

\section{Funding}

This study was funded by Practical Psychology Institute, LLC (COVID-19 related special research funds 2020.)

\section{Competing Interests}

The authors have declared that no competing interests exist.

\section{References}

1. Rajkumar RP. COVID-19 and mental health: A review of the existing literature. Asian J Psychiatr. 2020; 52: 102066.

2. Fitzpatrick KM, Harris C, Drawve G. Fear of COVID-19 and the mental health consequences in America. Psychol Trauma. 2020; 12: S17-S21.

3. Van Agteren J, Bartholomaeus J, Fassnacht DB, lasiello M, Ali K, Lo L, et al. Using internet-based psychological measurement to capture the deteriorating community mental health profile during COVID-19: Observational study. JMIR Mental Health. 2020; 7: e20696.

4. Li HY, Cao H, Leung DY, Mak YW. The psychological impacts of a COVID-19 outbreak on college students in China: A longitudinal study. Int J Environ Res Public Health. 2020; 17: 3933.

5. Chen L, Zhu H, Harshfield GA, Treiber FA, Pollock JS, Pollock D, et al. Serum 25-hydroxyvitamin $D$ concentrations are associated with mental health and psychosocial stress in young adults. Nutrients. 2020; 12: 1938.

6. Di Nicola M, Dattoli L, Moccia L, Pepe M, Janiri D, Fiorillo A, et al. Serum 25-hydroxyvitamin D levels and psychological distress symptoms in patients with affective disorders during the 
COVID-19 pandemic. Psychoneuroendocrinology. 2020; 122: 104869.

7. Ni MY, Yang L, Leung CM, Li N, Yao XI, Wang Y, et al. Mental health, risk factors, and social media use during the COVID-19 epidemic and cordon sanitaire among the community and health professionals in Wuhan, China: Cross-sectional survey. JMIR Mental Health. 2020; 7: e19009.

8. Cao W, Fang Z, Hou G, Han M, Xu X, Dong J, et al. The psychological impact of the COVID-19 epidemic on college students in China. Psychiatry Res. 2020; 287: 112934.

9. Wang H, Xia Q, Xiong Z, Li Z, Xiang W, Yuan Y, et al. The psychological distress and coping styles in the early stages of the 2019 coronavirus disease (COVID-19) epidemic in the general mainland Chinese population: A web-based survey. PLOS ONE. 2020; 15: e0233410.

10. Guo J, Feng XL, Wang XH, van IJzendoorn MH. Coping with COVID-19: Exposure to COVID-19 and negative impact on livelihood predict elevated mental health problems in Chinese adults. Int J Environ Res Public Health. 2020; 17: 3857.

11. Strickhouser JE, Zell E, Krizan Z. Does personality predict health and well-being? A metasynthesis. Health Psychol. 2017; 36: 797-810.

12. Moccia L, Janiri D, Pepe M, Dattoli L, Molinaro M, De Martin V, et al. Affective temperament, attachment style, and the psychological impact of the COVID-19 outbreak: An early report on the Italian general population. Brain Behav Immun. 2020; 87: 75-79.

13. Whitehead BR. COVID-19 as a stressor: Pandemic expectations, perceived stress, and negative affect in older adults. J Gerontol B. 2021; 76: e59-e64.

14. Yan L, Gan Y, Ding X, Wu J, Duan H. The relationship between perceived stress and emotional distress during the COVID-19 outbreak: Effects of boredom proneness and coping style. J Anxiety Disord. 2021; 77: 102328.

15. Kiuchi K, Kishi K, Araki K. A foundational assessment of the effects of the spread of COVID-19 virus infection and related activity restrictions on mental and physical health, psychological distress, and suicidal ideation in Japan. Asia Pac J Public Health. 2020; 32: 463-466.

16. Gosling SD, Rentfrow PJ, Swann Jr WB. A very brief measure of the Big-Five personality domains. J Res Pers. 2003; 37: 504-528.

17. Oshio $A$, Shingo $A B$, Cutrone P. Development, reliability, and validity of the Japanese version of ten item personality inventory (TIPI-J). Jpn J Pers. 2012; 21: 40-52.

18. I wasa H, Gondo Y, Masui Y, Inagaki H, Kawai C, Otsuka R, et al. Reliability and validity of the social support scale Japanese version: A study of middle-aged and older adults. J Health Welf Stat. 2007; 54: 26-33.

19. Zimet GD, Dahlem NW, Zimet SG, Farley GK. The multidimensional scale of perceived social support. J Pers Assess. 1988; 52: 30-41.

20. Ware J, Kosinski M, Dewey J, Gandek B. How to score and interpret single-item health status measures: A manual for users of the of the SF-8 health survey. Lincoln, RIQualityMetric Inc. Boston, MA: Health Assessment Lab; 2001.

21. Fukuhara S, Suzukamo Y. Manual of the SF-8 Japanese edition. Kyoto: Institute for Health Outcomes \& Process Evaluation Research; 2004. pp.7-31.

22. Kessler RC, Andrews G, Colpe LJ, Hiripi E, Mroczek DK, Normand SL, et al. Short screening scales to monitor population prevalences and trends in non-specific psychological distress. Psychol Med. 2002; 32: 959-976.

23. Furukawa TA, Kawakami N, Saitoh M, Ono Y, Nakane $\mathrm{Y}$, Nakamura $\mathrm{Y}$, et al. The performance of the Japanese version of the K6 and K10 in the world mental health survey Japan. Int J Methods 
Psychiatr Res. 2008; 17: 152-158.

24. National Information Center of Stress and Disaster Mental Health. 2010 national basic life survey special summary K6 [Internet]. 2010. Available from: https://saigaikokoro.ncnp.go.jp/k6.html.

25. Osman A, Bagge CL, Gutierrez PM, Konick LC, Kopper BA, Barrios FX. The Suicidal Behaviors Questionnaire-Revised (SBQ-R): Validation with clinical and nonclinical samples. Assessment. 2001; 8: 443-454.

26. Hu LT, Bentler PM. Cutoff criteria for fit indexes in covariance structure analysis: Conventional criteria versus new alternatives. Struct Equ Modeling. 1999; 6: 1-55.

27. Statistics Bureau of Japan. Labour force survey 2020 Feb statistical table I-2 [Internet]. 2020. Available from:

https://www.e-stat.go.jp/en/statsearch/files ?page $=1 \&$ layout $=$ datalist $\&$ touke $=00200531 \&$ tstat $=000000110001 \&$ cycle $=1 \&$ year $=$ 20200\&month $=11010302 \&$ tclass $1=000001040276 \&$ tclass $2=000001040283 \&$ tclass $3=0000010$ 40284\&result back=1\&tclass 4 val $=0$.

28. Klonsky ED, May AM, Saffer BY. Suicide, suicide attempts, and suicidal ideation. Annu Rev Clin Psychol. 2016; 12: 307-330.

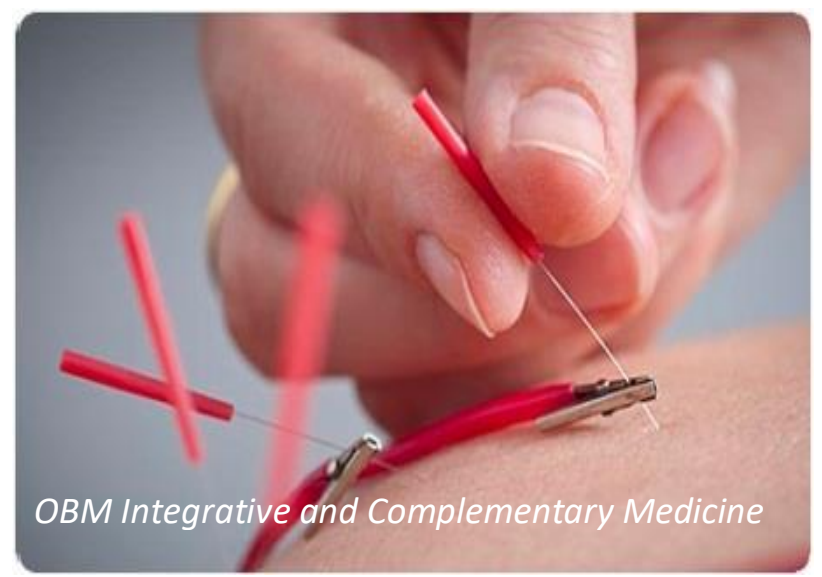

Enjoy OBM Integrative and Complementary Medicine by:

1. Submitting a manuscript

2. Joining in volunteer reviewer bank

3. Joining Editorial Board

4. Guest editing a special issue

For more details, please visit: http://www.lidsen.com/journals/icm 